\title{
IMPACT OF CHRONIC NOISE ON HIPPOCAMPAL MORPHOLOGY AND ITS FUNCTIONS IN WISTAR ALBINO RATS
}

\author{
ARCHANA ARJUNAN, RAVINDRAN RAJAN* \\ Department of Physiology, Dr. Arcot Lakshmanaswami Mudaliar Post Graduate Institute of Basic Medical Sciences, University of Madras, \\ Chennai, Tamil Nadu, India. Email: ravindarn89@gmail.com
}

Received: 07 October 2020, Revised and Accepted: 07 June 2021

\section{ABSTRACT}

Objective: The study aims to investigate the effect of chronic noise stress on hippocampal morphology and its functions in male Wistar albino rats.

Methods: Adult albino rats were randomly divided into two groups. Each group contained six animals. Rats exposed to chronic noise stress (100 dB/4 h-30 days) were compared with control animal and assessed for behavior using hole-board test, marble burying test, and morphology of hippocampus by histology.

Results: The rats exposed to chronic noise stress showed significance $(P<0.05)$ of behavioral alterations such as increased fear and anxiety, obsessivecompulsive behavior, enlarged lateral ventricle, and reduced hippocampal volume.

Conclusion: The results reported that chronic noise stress affects neurobehavioral due to reduced hippocampal volume.

Keywords: Anxiety, Fear, Hippocampus, Lateral ventricle, Noise, Obsessive-compulsive, Stress.

(c) 2021 The Authors. Published by Innovare Academic Sciences Pvt Ltd. This is an open access article under the CC BY license (http://creativecommons.org/ licenses/by/4.0/) DOI: http://dx.doi.org/10.22159/ajpcr.2021v14i7.39944. Journal homepage: https://innovareacademics.in/journals/index.php/ajpcr

\section{INTRODUCTION}

All living creatures depend on their environments for energy and materials that help in sustaining life. Although the environment sustains human life, it can also cause diseases [1]. The health impacts caused by excessive environmental noise are a growing concern. Noise exposure has been linked to a variety of health effects, including hearing loss, cardiovascular damage, cognitive impairment, and sleep disturbance, and annoyance [2]. Enormous studies have shown that noise above $90 \mathrm{~dB}$ increases the stress hormones and can cause a significant impact on human health. The brain is the target organ for corticosteroids [3] and the hippocampus is vulnerable to a number of insults and is prone to excitotoxic activity [4]. Receptors for glucocorticoids are found in the hippocampus, amygdala and frontal cortex, these three brain regions are involved in-memory processing, and emotional regulation $[3,5]$. From this, it was planned to study the effect of chronic noise on hippocampal functions in a noise-induced animal model.

\section{METHODS}

\section{Animals}

Experimental animals were all healthy and weighed about 140$180 \mathrm{~g}$. The animals were reared in the Animal House of the Institute, University of Madras, Taramani, Chennai, India, and all the animals were maintained under standard laboratory conditions housed at 3 per cage $(29 \mathrm{~cm} \times 22 \mathrm{~cm} \times 14 \mathrm{~cm})$ and constant ambient temperature with 12-h dark photoperiod; the rats were allowed free access to food and water. Appropriate ethical clearance was obtained for this work from the Institutional Animal Ethical Committee (IAEC no. 02/06/2019 dated $12 / 03 / 2019)$ prior to the experiments. Animals were divided into two groups, with six animals in each group.

- Group I - Control

- Group II - Chronic noise stress (100 d BA-4 h/30 days).

\section{Noise stress induction}

When noise exposure exceeds $100 \mathrm{dBA}$, it becomes a stressor. Noise was produced by two loudspeakers $(15 \mathrm{~W})$ driven by a white noise generator $(0-26 \mathrm{kHz})$, and they were installed $30 \mathrm{~cm}$ above the cage. The noise level was set at $100 \mathrm{~dB}$ uniformly throughout the cage and monitored by a sound-level meter D2023 (S.NO-F02199: Cygnet Systems, Gurgaon, Haryana, India). Animals were then exposed for $4 \mathrm{~h} / 30$ days. To avoid the influence of handling stress on evaluation, the effects of noise exposure in control animals were kept in the abovedescribed cage during the corresponding period of time, without noise stimulation [6].

\section{Marble burying test}

Marble burying test is used to assess the Obsessive-Compulsive behavior and is done using polycarbonate rat cages $(26 \mathrm{~cm} \times 48 \mathrm{~cm} \times$ $20 \mathrm{~cm}$ ) with fitted filter top covers, fresh, unscented mouse bedding material to each cage to a depth of $5 \mathrm{~cm}$ and level bedding surface by inserting another cage of the same size onto the surface of the bedding, and placed standard glass toy marbles (assorted styles and colors, $15 \mathrm{~mm}$ diameter, $5.2 \mathrm{~g}$ in weight) gently on the surface of the bedding in five rows of four marbles. Rats were placed into a corner of the cage containing marbles, with care taken to place the mouse on bedding as far from marbles as possible, and then the filter top was placed on top to cover the cage. Food and water were withhold during the test. The rats were allowed to remain undisturbed for $30 \mathrm{~min}$. Scoring was done when marble was buried and two-thirds of its surface area was covered by bedding. Average scores for the number of marbles buried for each rat were calculated [7].

\section{Hole board test}

The hole-board apparatus consisted of a wooden, gray box, measuring $68 \mathrm{~cm} \times 68 \mathrm{~cm}$. The walls were $40 \mathrm{~cm}$ high, and the box was raised $28 \mathrm{~cm}$ above the ground on a metal stand. Four holes ( $4 \mathrm{~cm}$ in diameter) were cut into the floor of the apparatus; each hole was $28 \mathrm{~cm}$ from a corner of the box along the diagonal from the corner to the center. The stand of the apparatus was open on all sides, allowing the floor or objects to be dimly lit. At the beginning of each trial, a patients was placed in one corner of the apparatus (always the corner closest to the door of the room), facing the center of the arena. Each trial lasted $10 \mathrm{~min}$. At the end of the trial, the patients was immediately placed into a carrying box 
and returned to the home cage. Between each trial, the floor and walls of the apparatus were cleaned using 70\% alcohol solution. During each 10-min trial, head-dip: the animal places its head into one of the holes, to a minimum depth such that the ears were in level with the floor of the apparatus (a new bout of head-dipping was recorded if the animal raised its head fully out of the hole before resuming); grooming, rearing, and fecal pellets' behavior patterns were recorded [8].

\section{Histology}

The rats were sacrificed by cervical dislocation. After sacrifice, the brain was rapidly removed, and the hippocampus was dissected on an ice-cold plate and the weight of the discrete region of the brain was measured. Another set of rats were subjected to transcardiac perfusion and fixed in $10 \%$ neutral formalin for $48 \mathrm{~h}$ solution followed by dehydration in ascending grades of alcohol, cleared, and then embedded in paraffin wax. Paraffin block $5 \mu \mathrm{m}$ thick coronal sections were obtained using a rotary microtome, and mounted on slides, followed by silver nitrate staining [9]. For Immunohistochemistry, the brains were postfixed with $10 \%$ formalin, embedded in paraffin, and cut into 10 -mm-thick sections. The sections were deparaffinized in xylene twice for $5 \mathrm{~min}$, rehydrated in a descending series of ethanol (99\%, 96\%, and 70\%), and followed by washes in distilled water. Antigen retrieval was achieved by heating the samples in a retrieval buffer with low $\mathrm{pH}$ of 6.2 at $110^{\circ} \mathrm{C}$ for $15 \mathrm{~min}$. Then, the sections were washed in wash buffer. The slides were incubated overnight with primary antibody (Wnt-3a [3A6]: sc136163, Santa Cruz Biotechnology, Inc (1: 100 Dilutions). After a rinse in PBS, the sections were incubated with rabbit biotinylated anti-goat IgG (1:200 Dilutions) for $1 \mathrm{~h}$, washed again with PBS, and incubated. All subsequent incubations were performed at room temperature and with DAB for $1 \mathrm{~h}$. The slides were counterstained with Mayer's Hematoxyline and mounted with DPX for examination. Photomicrographs were obtained using a Nikon Camera (Japan).

\section{Statistical analysis}

Data was presented in the form of the bar diagram with mean \pm SD. All data were analyzed with the Graphpad Prism 8. Statistical significance between the three groups was determined by $t$-test and $P<0.05$ was considered as statistically significant.

\section{RESULTS AND DISCUSSION}

Chronic stress induces reduction of the whole brain (Fig. 1) and hippocampal weight (Fig. 2) of the animals significantly when compared to the control animals. The number of marbles buried (Fig. 3) in the case of animals exposed to chronic noise were significantly greater when compared to the control animals. The number of head dipping (Fig. 4) reduced in chronic stress animals when compared to the control animals. In histological studies, sections obtained from the brain of chronic noise stress animals exhibited shrinking and enlargement of lateral ventricle when compared to the control brain (Figs. 5 and 6). Silver-staining methods are helpful for the histological identification of pathological deposits. In the control specimens, the brain neurons were stained yellowbrown, and in case of chronic stress exposed specimens, they were dark brown to black colored, the axon, cell body, neurofibrillary tangles, and neuropil threads were more readily apparent (Fig. 7). Hosseini-Sharifabad and Sabahi (2008) reported that the exposure of noise stress significantly reduced the hippocampal volume in all the layers of dentate gyrus and CA regions [10]. The hippocampal cells express the glucocorticoid receptors, they are the principal target sites for glucocorticoids which are the adrenocortical hormones secreted during stress [11]. Activation of GC receptors leads to the overproduction of ROS [12], and also increases oxidative damage to the protein due to the inhibiting activities of mitochondrial complex I and antioxidant enzyme SOD [13]. Conrad reported that chronic stress or prolonged exposure to glucocorticoids can compromise the hippocampus by producing dendritic retraction, a reversible form of plasticity that includes dendritic restructuring without irreversible cell death [14]. Reduced hippocampal volume causes behavioral changes such as obsessive-compulsive and increased fear and anxiety



Fig. 1: Weight of the whole brain

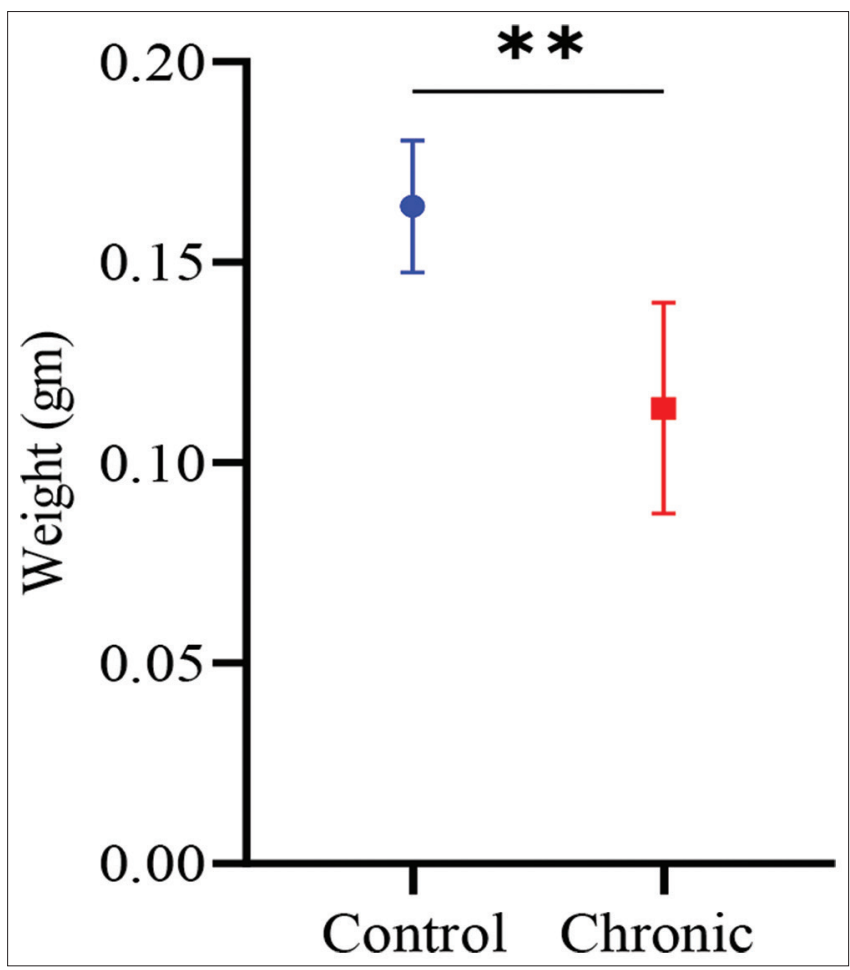

Fig. 2: Weight of hippocampus

in animals. The hippocampus has recently been identified to play a key role in the pathophysiology of adult obsessive-compulsive disorder (OCD) [15] and also Atamaca et al. (2008) reported that hippocampus, anterior cingulate, and basolateral amygdala interrelated together because of the fact that these structures have connections with the orbitofrontal cortex which may have a role in the pathophysiology of OCD and reduction of hippocampal volume to $12 \%$ in $\mathrm{AD}$ patients (Atmaca et al., 2008). 


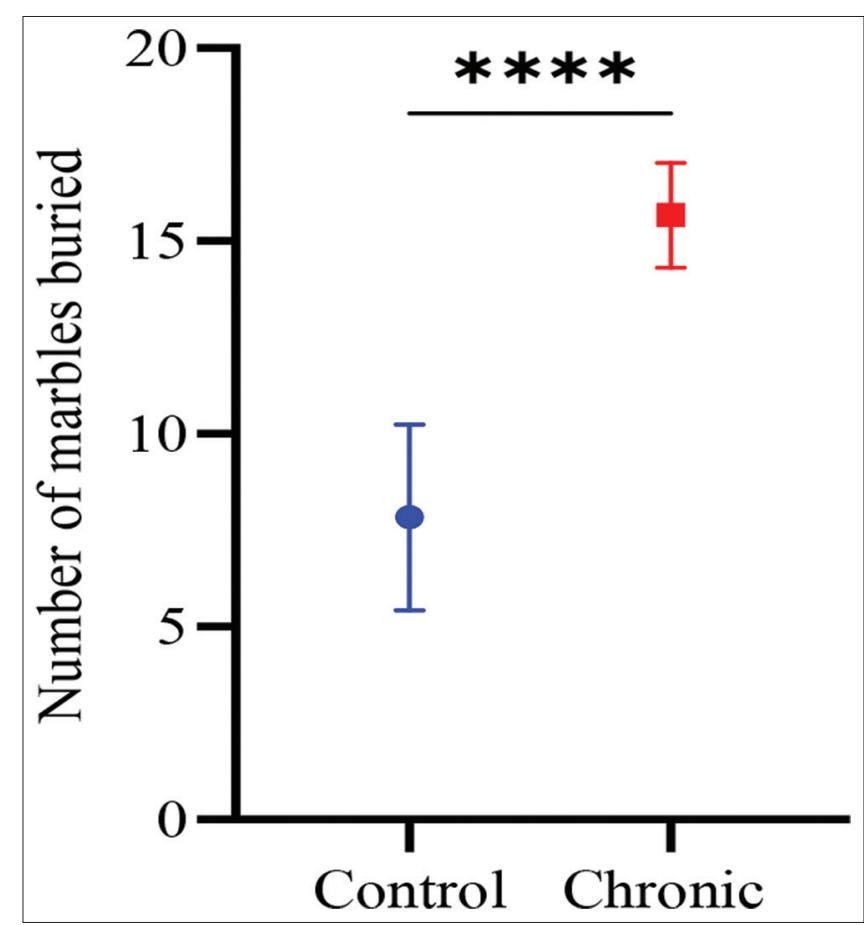

Fig. 3: Marble burying test

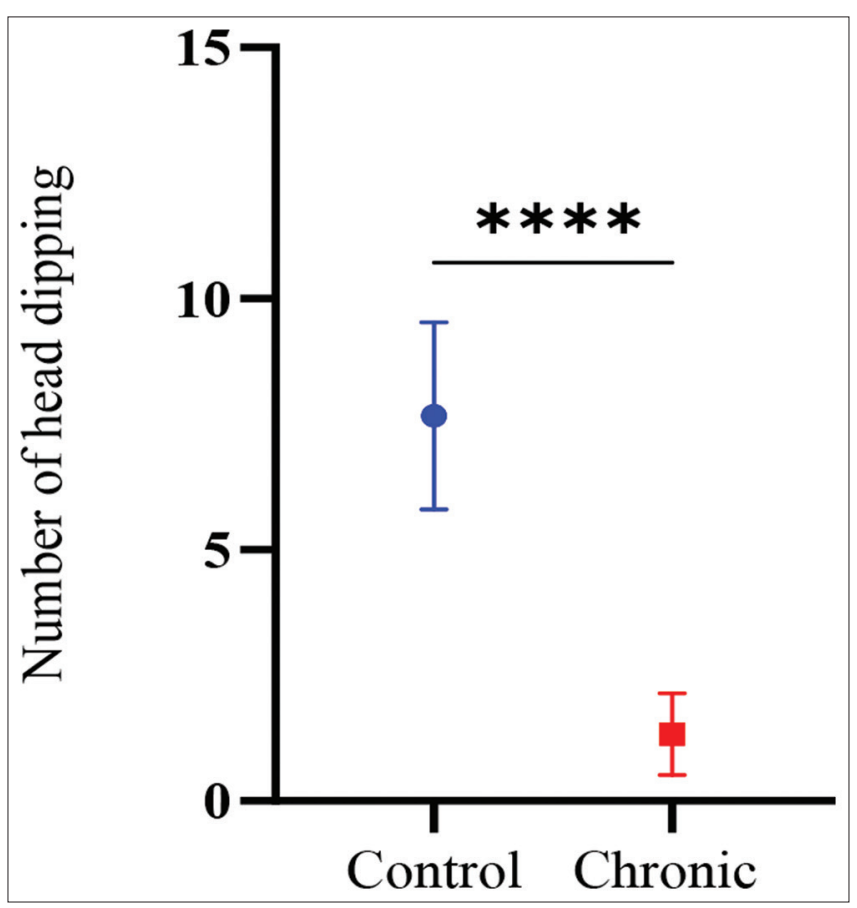

Fig. 4: Hole board test

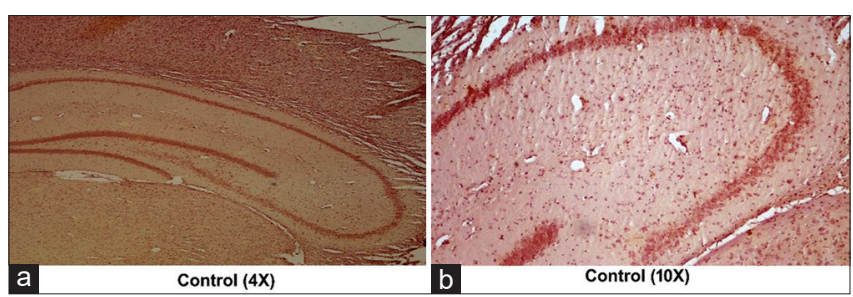

Fig. 5: (a and b) Shown control Hippocampus $\times 4$ and $\times 10$ magnification



Fig. 6: (a and b) Shown chronic noise stress-induced rat Hippocampus $\times 4$ and $\times 10$ magnifications. (ii) Shown shrunken hippocampus and enlarged space of lateral ventricle

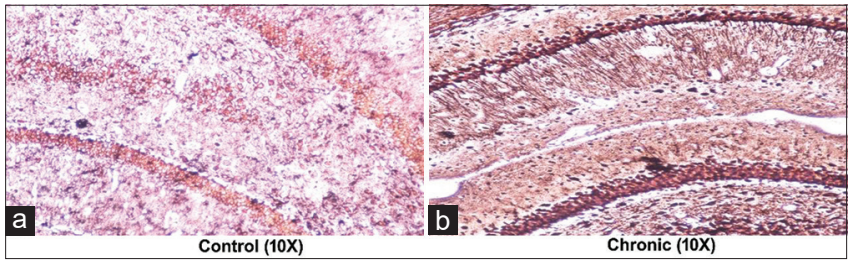

Fig. 7: (a and b) Shown silver nitrate staining of control and chronic noise with $\times 10$ magnification. (ii) Shown black brown cell bodies and axons

\section{CONCLUSION}

Noise beyond the bearable limit would make us feel upset and even get frustrated. When sound exceeds $90 \mathrm{~dB}$, it leads to deterioration of the health of an individual. The term "Noise stress" is a highly prevalent concept in the challenging and highly demanding fashionable society currently. In conclusion, our results confirmed that chronic noise stress led to a negative impact on the hippocampus and its functions in albino rats.

\section{ACKNOWLEDGMENT}

The authors are grateful to the University of Madras, for providing the infrastructure and TNSRC for provided funding to conduct the research. The authors would like to acknowledge Dhiraj Kumar Sah, Research Scholar, Department of Biomedical Sciences, Chonnam National University, South Korea, for helping out English and the final correction of the manuscript.

\section{AUTHORS CONTRIBUTION}

The corresponding author has designed the work and critical revision of the manuscript. The first author carried out behavioral assessment and paper writing.

\section{CONFLICT OF INTERST}

The authors declare that they no conflicts of interest concerning this research article.

\section{FUNDING}

This research received specific grant from TNSRC (2018-2019).

\section{REFERENCES}

1. Atmaca M, Yildirim H, Ozdemir H, Ozler S, Kara B, Ozler Z, et al. Hippocampus and amygdalar volumes in patients with refractory obsessive-compulsive disorder. Prog Neuropsychopharmacol Biol Psychiatry 2008;32:1283-6.

2. Salter CM, Ahn R, Yasin F, Hines R, Kornfield L, Salter EC, et al. Community noise, urbanization, and global health: Problems and solutions. In: Innovating for Healthy Urbanization, 2015. Doi: 10.1007/978-1-4899-7597-3 8

3. Krugers HJ, Lucassen PJ, Karst H, Joëls M. Chronic stress effects on hippocampal structure and synaptic function: Relevance for depression and normalization by anti-glucocorticoid treatment, Front. Synaptic 
Neurosci 2010;2010:24

4. Wible CG. Hippocampal physiology, structure and function and the neuroscience of schizophrenia: A unified account of declarative memory deficits, working memory deficits and schizophrenic symptoms, Behav Sci (Basel) 2013;2013:3020298

5. McEwen BS, Morrison JH. The brain on stress: Vulnerability and plasticity of the prefrontal cortex over the life course. Neuron 2013;79:16-29.

6. Arjunan A, Rajan R, Effect of noise stress-induced neurobehavioral changes on wistar albino rats. Asian J Pharm Clin Res 2019;12:34843.

7. Angoa-Pérez M, Kane MJ, Briggs DI, Francescutti DM, Kuhn DM. Marble burying and nestlet shredding as tests of repetitive, compulsivelike behaviors in mice. J Vis Exp 2013;82:50978.

8. Brown GR, Nemes C. The exploratory behaviour of rats in the holeboard apparatus: Is head-dipping a valid measure of neophilia? Behav Processes 2008;78:442-8.

9. Aboulaich N Silver staining. BioProtocol 2011;1:e26.

10. Hosseini-Sharifabad M, Sabahi A. Exposure to chronic noise reduces the volume of hippocampal subregions in rats. Iran J Basic Med Sci 2008;11:18-24.

11. Behl C, Lezoualc'h F, Trapp T, Widmann M, Skutella T, Holsboer F. Glucocorticoids enhance oxidative stress-induced cell death in hippocampal neurons in vitro. Endocrinology 1997;138:101-6.

12. You JM, Yun SJ, Nam KN, Kang C, Won R, Lee EH. Mechanism of glucocorticoid-induced oxidative stress in rat hippocampal slice cultures. Can J Physiol Pharmacol 2009;87:440-7.

13. Tang VM, Young AH, Tan H, Beasley C, Wang JH. Glucocorticoids increase protein carbonylation and mitochondrial dysfunction. Horm Metab Res 2013;45:709-15.

14. Conrad CD. Chronic stress-induced hippocampal vulnerability: The glucocorticoid vulnerability hypothesis. Rev Neurosci 2008;19:395-411.

15. Reess TJ, Rus OG, Gürsel DA, Schmitz-Koep B, Wagner G, Berberich $\mathrm{G}$, et al. Association between hippocampus volume and symptom profiles in obsessive-compulsive disorder. NeuroImage Clin 2018;17:474-80. 